\title{
The Emerging Use of IMRT for Treatment of Cervical Cancer
}

\author{
Christopher Loiselle, MD, and Wui-Jin Koh, MD, Seattle, Washington
}

Key Words

IMRT, cervix cancer, cervical cancer, radiation therapy, radiotherapy

\begin{abstract}
Radiation therapy plays an important role in both the definitive and adjuvant treatment of patients with cervical cancer. However, although radiation therapy is effective in controlling tumor growth, associated acute and chronic adverse effects are well known. Intensity-modulated radiation therapy (IMRT) is increasingly being used to treat cervical cancer and has the potential to improve the therapeutic ratio because of its ability to escalate dose to cancer targets while sparing adjacent healthy tissue. Multiple dosimetric studies were initially performed, establishing the conceptual feasibility of IMRT in patients with cervical cancer. Subsequent early reported series of patients treated with IMRT showed dosimetric and clinical benefits, with reduction in acute gastrointestinal and hematologic toxicity compared with historic controls, particularly in the posthysterectomy setting. Consensus is evolving regarding the use of IMRT in treating cervical cancer, particularly in the posthysterectomy setting, and for dose escalation to para-aortic nodes and bulky sidewall disease. Target delineation in the context of internal organ motion and tumor shrinkage during a course of fractionated external-beam radiotherapy remains an area of active investigation. IMRT in treating cervical cancer in
\end{abstract}

From the Department of Radiation Oncology, University of Washington Medical Center and Seattle Cancer Care Alliance, Seattle, Washington. Submitted September 9, 2010; accepted for publication October 30, 2010. Correspondence: Wui-Jin Koh, MD, Department of Radiation Oncology, University of Washington Medical Center, Seattle Cancer Care Alliance, 825 Eastlake Ave E., G1101, Seattle, WA 98109-1023.

E-mail: wkoh@u.washington.edu

\section{EDITOR}

Kerrin M. Green, MA, Assistant Managing Editor, Journal of the National Comprehensive Cancer Network

Disclosure: Kerrin M. Green, MA, has disclosed no relevant financial relationships. the setting of an intact uterus remains in its nascent stage and should be used judiciously only within clinical trials. Although not a routine substitute for brachytherapy, it may be considered as a boost for highly selected patients who are not brachytherapy candidates. (JNCCN 2010;8:1425-1434)

\section{Medscape: Continuing Medical Education Online}

\section{Accreditation Statement}

This activity has been planned and implemented in accordance with the Essential Areas and policies of the Accreditation Council for Continuing Medical Education through the joint sponsorship of Medscape, LLC and JNCCN - The Journal of the National Comprehensive Cancer Network. Medscape, LLC is accredited by the ACCME to provide continuing medical education for physicians. Medscape, LLC designates this educational activity for a maximum of 1.00 AMA PRA Category 1 Credits $^{\mathrm{TM}}$. Physicians should only claim credit commensurate with the extent of their participation in the activity. All other clinicians completing this activity will be issued a certificate of participation. To participate in this journal CME activity: (1) review the learning objectives and author disclosures; (2) study the education content; (3) take the post-test and/ or complete the evaluation at www.medscapecme.com/journal/ jnccn; (4) view/print certificate.

\section{AUTHORS AND CREDENTIALS}

Christopher Loiselle, MD, Department of Radiation Oncology University of Washington Medical Center; Seattle Cancer Care Alliance, Seattle, Washington

Disclosure: Christopher Loiselle, MD, has disclosed no relevant financial relationships.

Wui-Jin Koh, MD, Department of Radiation Oncology, University of Washington Medical Center; Seattle Cancer Care Alliance, Seattle, Washington

Disclosure: Wui-Jin Koh, MD, has disclosed no relevant financial relationships.

\section{CME AUTHOR}

Laurie Barclay, MD, Freelance writer and reviewer, Medscape, LLC Disclosure: Laurie Barclay, MD, has disclosed no relevant financial relationships. 
Loiselle and Koh

\section{Learning Objectives}

Upon completion of this activity, participants will be able to:

- Describe potential advantages and disadvantages of pelvic IMRT

- Describe technical strategies that may improve the therapeutic ratio of IMRT

- Describe clinical scenarios for which IMRT is indicated and those for which it is less useful

In 2010, the American Cancer Society (ACS) has estimated that 12,200 cases of uterine cervical cancer will be diagnosed and 4210 deaths will occur from the disease in the United States. ${ }^{1}$ Worldwide, the most recent yearly ACS estimate of cervical cancer cases was 555,094, with an associated 309,808 deaths. ${ }^{2}$ Among women globally, cervical cancer is second only to breast cancer in incidence, and third after breast and lung cancers with regard to mortality.

For many of these women, radiation therapy plays an important role in both the definitive and adjuvant (i.e., posthysterectomy) treatment setting. Definitive pelvic radiotherapy with concurrent platinum-based chemotherapy and brachytherapy boost has been established as the standard of care in locally advanced disease. In 1999, the publication of 5 randomized trials ${ }^{3-7}$ led to the February 1999 NCI recommendation to consider concurrent platinumbased chemoradiation as primary therapy for patients with locally advanced cervical cancer. In the setting of clinical early-stage disease after radical hysterectomy, adjuvant pelvic radiotherapy is recommended, depending on the extent of cervical stromal invasion, lymphovascular space invasion, and tumor size, although adjuvant chemoradiation is recommended in the setting of positive lymph nodes after radical hysterectomy, involved parametria, or positive surgical margins.

Although radiation therapy maintains an effective track record in controlling locoregional tumors, associated acute and chronic adverse effects are wellknown. Nausea, vomiting, diarrhea, irritation of the urinary tract, and bone marrow suppression are common side effects experienced by patients undergoing pelvic radiotherapy. Furthermore, these side effects are enhanced with the administration of concurrent chemotherapy. In the acute setting of chemoradiation, low-grade genitourinary, gastrointestinal, and hematologic toxicity have been reported, with respective rates of $17.5 \%, 45.2 \%$, and $53.3 \%$. High- grade acute effects ( 3 or 4 ) are less common, but rates of $1.5 \%$ genitourinary, $8 \%$ gastrointestinal, and $27.6 \%$ hematologic toxicity have been noted. ${ }^{8}$

In the longer term, anatomic and physiologic alterations, such as obstruction and strictures, fistulization, pelvic insufficiency fracture, soft tissue fibrosis or necrosis, and lymphedema, are potential causes of severe morbidity. Although less well documented than acute toxicities, incidence of late grade 3 and 4 complications have ranged from $6 \%$ to $23.3 \%$ of patients. ${ }^{8}$ Most clinical trials have historically underemphasized late low-grade (i.e., grade 1 and 2) effects, but with recent increased emphasis on long-term quality of life, experts have recognized that this low-grade late toxicity (e.g., anal leakage) can be prevalent. ${ }^{9}$

The external beam component of radiation therapy has evolved dramatically in the past 2 decades and is now capable of differentially targeting tumor and normal tissues in the pelvis. Traditional anteroposterior and " 4 field box" radiation therapy plans based on bony landmarks have been shown to inadequately address intended targets in many cases, ${ }^{10}$ leading to the widespread implementation of 3-dimensional conformal treatment planning, facilitated with modern cross-sectional imaging.

Intensity-modulated radiation therapy (IMRT) has potential to further improve the therapeutic ratio of external-beam radiotherapy. The improved conformality achievable with IMRT can potentially mitigate adverse effects and, in some clinical scenarios, allow dose to be escalated to target volumes to optimize tumor control. Traditionally, whole-pelvic doses are limited to 45 to 50 Gy, primarily as a result of small bowel tolerance. In this dose range, greater volumetric sparing of small bowel with IMRT will reduce the risk of acute and late toxicity. Conversely, IMRT may also permit selective boosting of gross disease sites to higher tumoricidal doses, without a corresponding increase in small bowel dose.

The use of IMRT has increased dramatically over the past decade. The percentage of practicing radiation oncologists using IMRT increased from $32 \%$ in 2002 to $73 \%$ in $2004 .{ }^{11,12}$ In the upper pelvis, IMRT increases sparing of the small bowel (particularly in the posthysterectomy setting) and bone marrow of the iliac wings (Figure 1). In the low pelvis, IMRT better spares the bladder, rectum, and femoral heads (Figure 2). The authors have used IMRT in selected posthysterectomy pelvic irradiation cases, in dose escalation for grossly 


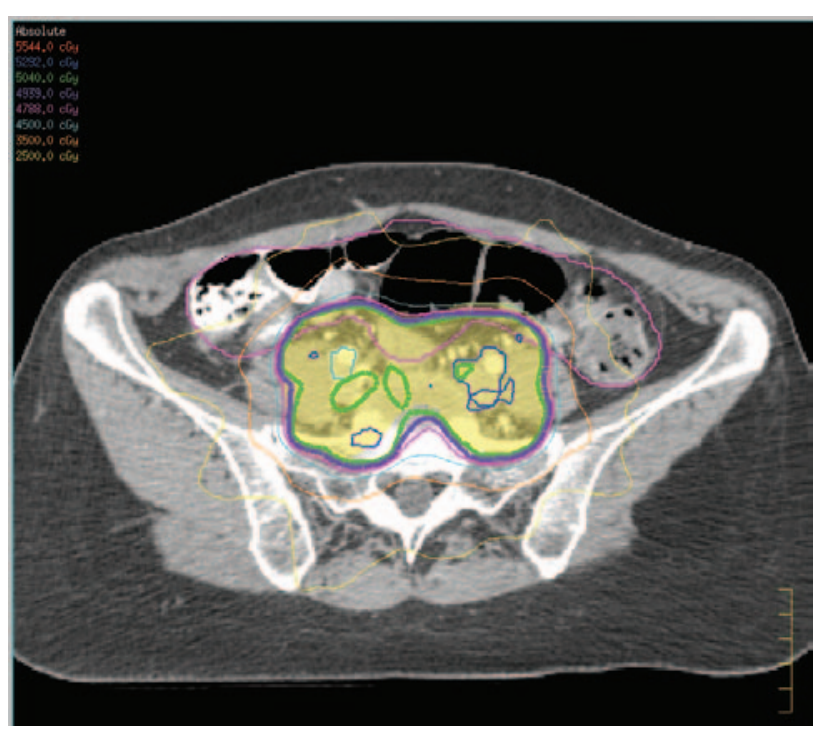

Figure 1 Upper pelvis axial CT planning image for an intensity-modulated radiation therapy (IMRT) dose plan in a patient status post radical hysterectomy with a positive resected pelvic lymph node undergoing concurrent chemoradiation. Nodal planning volume and small bowel are outlined. The prescription 5040 cGy representing the $100 \%$ isodose as well as the $110 \%, 105 \%, 98 \%, 95 \%, 89 \%, 69 \%$, and $50 \%$ isodose lines are shown.

positive para-aortic lymph nodes, in patients unfit for brachytherapy, and in reirradiation cases.

\section{Technical Factors}

IMRT has been made possible through advancements in high-definition cross-sectional imaging and dose algorithm computing, which were first pioneered and widely applied in the setting of head and neck, and prostate cancers. The use of IMRT requires precise delineation of target volumes and organs at risk (OARs). Dose is prescribed not to a single point or fixed volume but to a "best fit" based on dose-volume objectives. Dose to a cancer-bearing region is understood and reported in terms of gross target volume (GTV), clinical target volume $(\mathrm{CTV}=\mathrm{GTV}+$ potential microscopic regions at risk), and planning target volume (PTV $=\mathrm{CTV}+$ margins for patient and organ movement and daily setup reproducibility). The prescription is optimized based on physician defined dose-volume constraints, specified for both target volumes and OARs. Significantly more time and resources by physicians, physicists, and technical staff are required for IMRT planning and delivery, compared with conventional 3-dimensional treatment approaches. Tumor target volumes are delineated by

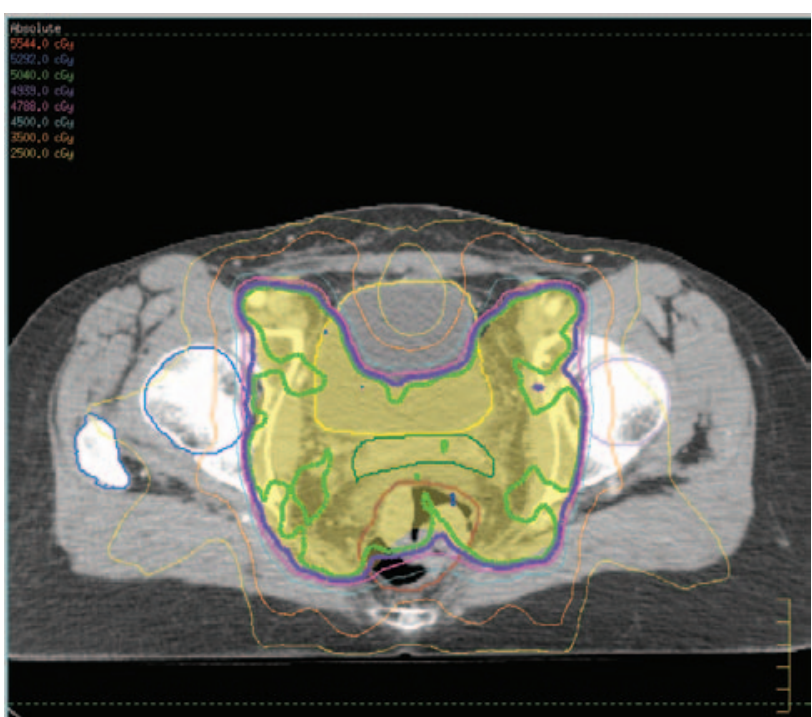

Figure 2 Lower pelvis axial CT planning image for an intensity-modulated radiation therapy (IMRT) dose plan in a patient status post radical hysterectomy with a positive resected pelvic lymph node undergoing concurrent chemoradiation. Planning target volume, rectum, vagina, and bladder are outlined. The prescription $5040 \mathrm{cGy}$ representing the $100 \%$ isodose as well as the $110 \%, 105 \%, 98 \%, 95 \%, 89 \%, 69 \%$, and $50 \%$ isodose lines are shown.

correlation of imaging and physical examination findings, supplemented with a clear understanding of locoregional pathways of spread, and are often expanded asymmetrically based on the juxtaposition of adjacent normal tissues and anatomical barriers to tumor extension. Individual organs at risk within the planned irradiated volume must be contoured, and appropriate dosevolume constraints determined.

A "good" IMRT plan appropriately optimizes target volume and OAR constraints to maximize the therapeutic ratio, through multiple iterations using a computer inverse algorithm and through multistep physician feedback after review of computer-generated isodose plans and dose-volume histograms. ${ }^{13}$ Adhering to the complex treatment and dosimetric plans developed, dosing and linear accelerator setup specifications are then controlled with sophisticated computer algorithms, in which the target volume achieves its ultimate intended dose through the integration of multiple fragmental portions of radiation exposure. At no single timepoint is the entire target volume delivered at a homogenous dose. With the numerous beam segments and subsegments used for IMRT delivery, obtaining portal imaging that shows the entire irradiated volume is impractical or impos- 
Loiselle and Koh

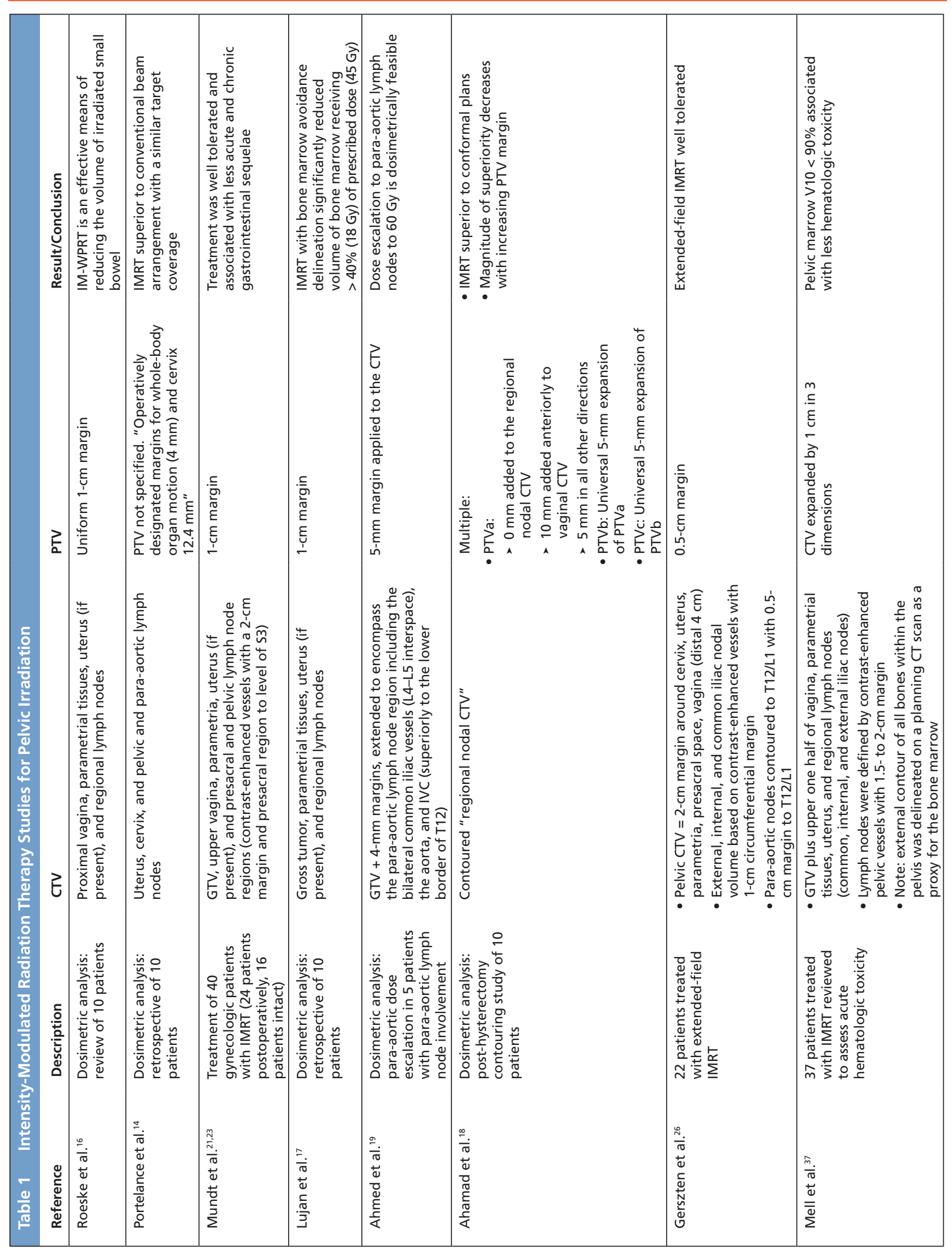




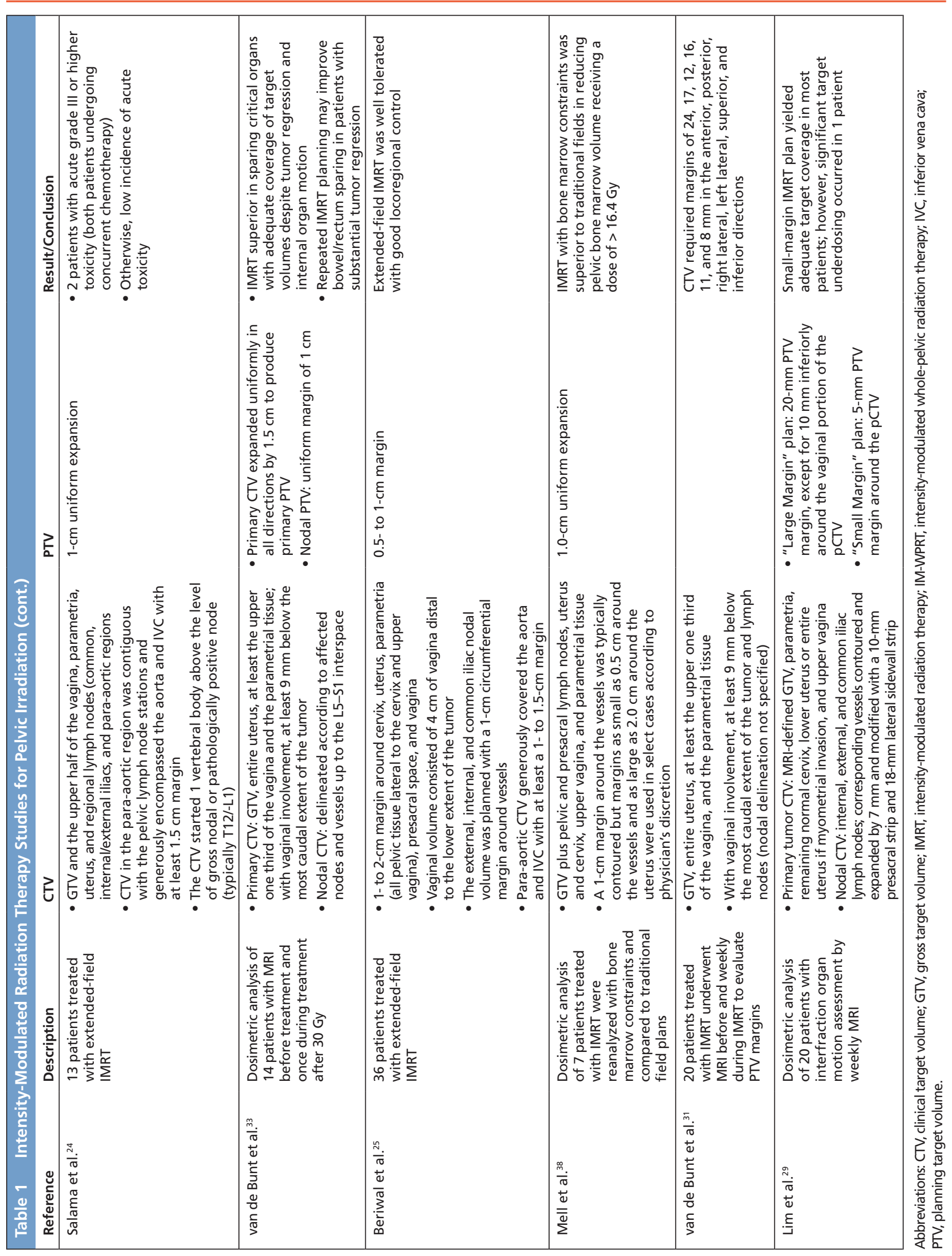


Loiselle and Koh

sible. Therefore, any clinical IMRT delivery, meticulous quality assurance measurements using diodes and ion chambers must be performed to calibrate and confirm each patient plan.

\section{Dosimetric Feasibility and Clinical Applicability}

Dosimetric analyses and clinical patient series have examined the potential of IMRT to limit adverse effects associated with pelvic radiotherapy (Table 1). Dosimetric studies initially set the foundation for IMRT use, showing substantially less volume of small bowel, bladder, rectum, and bone marrow receiving a given prescription pelvic dose than with conventional external-beam plans, ${ }^{14-18}$ and further extrapolated benefits of normal tissue sparing while allowing dose escalation to the tumor in the setting of positive para-aortic lymph nodes. ${ }^{19}$

Mundt et al..$^{20,21}$ reported the first clinical series of 40 patients treated with whole-pelvis IMRT in 2001, most of whom were posthysterectomy, compared with 35 historic controls treated with conventional fields. Grade II acute gastrointestinal toxicity was less common with IMRT ( $60 \%$ vs. $91 \% ; P=.002$ ), with $75 \%$ of patients requiring no or only infrequent antidiarrheal medications compared with $34 \%$ of patients treated with traditional fields $(P=.001)$. Acute hematologic toxicity in patients treated concurrently with chemotherapy was significantly less, and chemotherapy was withheld or delayed less often in patients treated with IMRT ( $40 \%$ vs. $12.5 \%$; $P=.06$ ), attributed to less iliac crest bone marrow volume being irradiated. ${ }^{22}$ Chronic gastrointestinal toxicity 1 year after treatment was also decreased with IMRT. ${ }^{23}$

Other published studies have described the treatment of patients with extended-field IMRT for dose escalation to para-aortic nodes. ${ }^{24-26}$ Salama et al. ${ }^{24}$ reported on toxicity in 13 patients treated with extended-field IMRT for gynecologic malignancies (11 of whom were posthysterectomy). Patients received 45 Gy in 1.8-Gy daily fractions, with an additional 9-Gy boost to bulky nodes, in the para-aortics or pelvis. Two patients developed acute grade III toxicity, both of whom were treated with concurrent weekly cisplatin chemotherapy. Two patients experienced late toxicity in the context of their overall therapeutic course: 1 had undergone multiple prior abdominal surgeries and experienced a small bowel obstruc- tion requiring partial colectomy, and the other had grossly involved common iliac nodes and developed bilateral lower-extremity edema. ${ }^{24}$

Gerszten et al. ${ }^{26}$ similarly showed feasibility of extended-field coverage with IMRT, using a simultaneous integrated boost technique involving $45 \mathrm{~Gy}$ delivered in 25 fractions to the entire target volume, with simultaneous boost to 55 Gy to involved nodes in the same number of fractions. No patients experienced acute grade III or IV toxicity.

Beriwal et al. ${ }^{25}$ reported on 36 patients treated with extended-field IMRT for cervical carcinoma. Para-aortic nodes were treated to the superior L1 vertebral border. The treatment was found to be well tolerated, with acute grade III or greater gastrointestinal and genitourinary toxicity seen in only a single patient each. Although ultimate distant failure occurred in 9 patients, only 2 experienced relapse within the irradiated volume (1 pelvic, 1 para-aortic).

Overall, these studies have shown dosimetric and suggested clinical benefits with pelvic IMRT, with reduced acute gastrointestinal and hematologic toxicity and decreased long-term gastrointestinal toxicity. IMRT also enabled better tolerance of concurrent chemotherapy. Furthermore, several patient series have shown the feasibility of IMRT in achieving tolerable dose escalation to involved para-aortic lymph nodes.

\section{Challenges}

Target delineation in the context of internal organ motion and tumor shrinkage during a course of fractionated external-beam radiotherapy treatment poses challenges to treatment with IMRT, with the implied limited target margins. The bladder, rectum, and other bowels are dynamic and mobile structures in the pelvis in direct proximity to target structures. Bladder and rectal filling can vary dose distribution to these normal structures and alter GTV position. 27,28 Tumor deformation can alter the arrangement of surrounding normal tissues. Tumor regression during treatment is a fortunate, though complex, reality and renders target definition based on a single preradiation CT scan less dependable.

Serial MRI has been used to characterize motion of the uterus and cervix. ${ }^{29}$ One dosimetric study found that margins of $4 \mathrm{~cm}$ at the fundus and 1.5 $\mathrm{cm}$ at the cervical os were necessary to encompass $90 \%$ of interscan motion. ${ }^{30}$ Another article sug- 
gested that 2-cm PTV expansions led to statistically similar coverage of targets compared with a " 4 field box"; however, significant underdosing occurred in 1 patient who displayed excessive internal target movement. In a study evaluating patients with MRI before and weekly during IMRT, additional margins were required to accommodate $95 \%$ of movement in multiple dimensions. ${ }^{31}$

Rapid involution and mobility of carcinoma of the cervix in response to radiotherapy has been described in multiple studies. Lee et al..$^{32}$ described 50\% tumor regression at approximately 21 days at a median radiation dose of $30.8 \mathrm{~Gy}$. Van de Bunt et al..$^{33}$ reported that after 30 Gy, the primary GTV decreased an average of $46 \%$. Replanning with IMRT significantly diminished the treated bowel volume if the primary GTV decreased by $30 \mathrm{~cm}^{3} .^{33}$ Beadle et al..$^{34}$ showed mean volume reduction of $62.3 \%$ after 45 Gy.

Altogether, although IMRT offers potentially superior planned dose distribution, its superiority remains dependent on accurate delivery of fields and avoidance of possible heterogenous hot or cold spots within the irradiated volume. Further adding complexity is the dynamic environment of the pelvis; patient movement, cancer target movement, normal structure movement, and tumor shrinkage during treatment are all important factors to consider when delineating treatment plan specifications. Planning appropriate target margins given internal target motion and variability is a challenge, and remains particularly undefined in the intact uterus setting, where the fundus movement may be appreciable and gross temporal tumor regression significant. Image-guided radiation therapy (IGRT), including the use of regular cone-beam CT, may help improve and verify localization and positioning during a course of IMRT.

\section{Cooperative Group Trials}

The Radiation Therapy Oncology Group (RTOG) has completed accrual for RTOG-0418, a phase II trial evaluating the use of pelvic IMRT in the posthysterectomy setting for patients with endometrial or cervical carcinoma. Objectives for this study include testing the hypothetical reduction in shortterm bowel injury and estimating the rates of locoregional control, disease-free survival, overall survival, and chemotherapy compliance. Furthermore, the trial was designed to evaluate IMRT feasibility, re- producibility, and ability to assess dosimetry plans in clinical trials across multiple institutions. The vaginal planning target volume was derived from an internal target volume (ITV) with a 7-mm expansion. The ITV was based on fused full and empty bladder scans, allowing for a composite vagina and paravaginal soft tissue volume, taking into account the variation of the target caused by bladder filling and motion. The nodal CTV included internal, external, and common iliac lymph nodes. If the cervix was involved, presacral lymph nodes were included down to S3. Preliminary analysis showed general feasibility for the use of IMRT in multi-institutional trials when stringent guidelines are specified..$^{35}$

Cervical cancer clinical trials continue to use IMRT. The RTOG 0724 trial evaluating adjuvant therapy for lymph node-positive cervical cancer and Gynecologic Oncology Group (GOG) 263 for lymph node-negative cervical cancer allow IMRT, again with carefully detailed guidelines of application. Both the RTOG and GOG protocols specify a vaginal ITV as a composite volume derived from fused full and empty bladder scans.

Consistency in volume and contouring definitions is essential among centers using IMRT in multicenter trials. The RTOG atlas "Consensus Guidelines for the Delineation of the CTV in the Postoperative Pelvic Radiotherapy of Endometrial and Cervical Cancer" for posthysterectomy contouring is available at http://www.rtog.org/pdf_document/GYN-Atlas.pdf.

The role of IMRT in treating intact cervical cancer remains even more nascent and investigational, and should be performed only in prospective clinical trials. Target mobility and tumor deformation/regression during a course of radiotherapy are of greater concern in intact cancer cases than in the posthysterectomy setting. A preliminary attempt at target volume consensus in intact cervical cancer (based on a single case study) was recently published. ${ }^{36} \mathrm{MR}$ imaging with fusion of the T2-weighted axial images to the planning CT is strongly recommended. The CTV includes the GTV, remaining cervix, entire uterus, parametria, ovaries, vaginal tissues, and nodal region, varyingly comprised of the common, internal iliac, external iliac, obturator, presacral, and para-aortic nodes, depending on the extent of disease. PTV margins of 1.5 to $2 \mathrm{~cm}$ around the primary CTV are recommended if good-quality daily soft tis- 
Loiselle and Koh

sue verification is available and a $7-\mathrm{mm}$ margin is present around the nodal CTV. If daily verification with bone landmarks is performed instead, more generous PTV margins are required. Based on this target delineation analysis, phase II trials for IMRT in intact cervical cancer have been proposed.

\section{Conclusions}

Consensus is evolving, founded in a decade of published studies and evidenced by its adoption in cooperative group trials, that IMRT has clear applicability in cervical cancer, particularly in the posthysterectomy setting. IMRT offers distinct dosimetric advantages over traditional 2 - and 3-dimensional planning techniques with regard to sparing normal tissues adjacent to cancer targets. The use of IMRT in a multicenter cooperative group trial has been tested and found to be feasible in posthysterectomy cases. The hope is that further study of IMRT will continue to confirm that patients undergoing surgery followed by adjuvant radiation with or without chemotherapy will experience excellent locoregional cancer control and decreased short- and long-term toxicity. Furthermore, IMRT allows dose escalation to para-aortic lymph nodes and bulky sidewall disease, and may be useful in reirradiation cases. IMRT may also be considered as a boost to primary disease in patients who are not brachytherapy candidates. However, application of IMRT for cervical cancer in the setting of an intact uterus and cervix remains in its nascent stage and should not be accepted as a routine substitute for brachytherapy.

IMRT remains a complex and heterogeneous technique. Treatment planning options, including the number of incident beams, beam angles, target volumes, and normal tissue constraints, render it a flexible and powerful tool. However, it is also subject to inadvertent inadequate dose coverage or overdose caused by target motion or suboptimal target delineation. Furthermore, with implementation of inverse planning techniques, the physician designates "intuitive" dose delivery control to the computer, thus requiring rigorous treatment planning and delivery quality assurance procedures. High-quality imaging and image interpretation are imperative for highfidelity treatment delivery. The intra- and interfraction motion described earlier suggest a role for a daily 3-dimensional image-guided setup, particularly in emerging areas of study for treating the intact cervix.

The way forward is through continued evolution within clinical trials. With regard to cancer control, normal tissue impact, and long-term assessment of quality of life, further prospective multi-institutional studies will help establish the ultimate role of IMRT in cervical cancer management.

\section{References}

1. American Cancer Society. Cancer Facts \& Figures 2010. Available at: http://www.cancer.org/acs/groups/content/@ epidemiologysurveilance/documents/document/acspc-026238.pdf Accessed November 3, 2010.

2. American Cancer Society. Global Cancer Facts \& Figures 2007. Available at: http://www.cancer.org/acs/groups/content/@nho/ documents/document/globalfactsandfigures 2007 rev2p.pdf. Accessed November 4, 2010.

3. Keys HM, Bundy BN, Stehman FB, et al. Cisplatin, radiation, and adjuvant hysterectomy compared with radiation and adjuvant hysterectomy for bulky stage IB cervical carcinoma. N Engl J Med 1999;340:1154-1161.

4. Morris M, Eifel PJ, Lu J, et al. Pelvic radiation with concurrent chemotherapy compared with pelvic and para-aortic radiation for high-risk cervical cancer. N Engl J Med 1999;340:1137-1143.

5. Peters WA III, Liu PY, Barrett RJ II, et al. Concurrent chemotherapy and pelvic radiation therapy compared with pelvic radiation therapy alone as adjuvant therapy after radical surgery in high-risk early-stage cancer of the cervix. J Clin Oncol 2000;18:1606-1613.

6. Rose PG, Bundy BN, Watkins EB, et al. Concurrent cisplatinbased radiotherapy and chemotherapy for locally advanced cervical cancer. N Engl J Med 1999;340:1144-1153.

7. Whitney CW, Sause W, Bundy BN, et al. Randomized comparison of fluorouracil plus cisplatin versus hydroxyurea as an adjunct to radiation therapy in stage IIB-IVA carcinoma of the cervix with negative para-aortic lymph nodes: a Gynecologic Oncology Group and Southwest Oncology Group study. J Clin Oncol 1999;17:13391348.

8. Kirwan JM, Symonds P, Green JA, et al. A systematic review of acute and late toxicity of concomitant chemoradiation for cervical cancer. Radiother Oncol 2003;68:217-226.

9. Nout RA, Putter H, Jurgenliemk-Schulz IM, et al. Quality of life after pelvic radiotherapy or vaginal brachytherapy for endometrial cancer: first results of the randomized PORTEC-2 trial. J Clin Oncol 2009;27:3547-3556.

10. Zunino $S$, Rosato $O$, Lucino $S$, et al. Anatomic study of the pelvis in carcinoma of the uterine cervix as related to the box technique. Int J Radiat Oncol Biol Phys 1999;44:53-59.

11. Mell LK, Mehrotra AK, Mundt AJ. Intensity-modulated radiation therapy use in the U.S., 2004. Cancer 2005;104:1296-1303.

12. Mell LK, Roeske JC, Mundt AJ. A survey of intensity-modulated radiation therapy use in the United States. Cancer 2003;98:204211.

13. Fiorino C, Valdagni R, Rancati $T$, et al. Dose-volume effects for normal tissues in external radiotherapy: pelvis. Radiother Oncol 2009;93:153-167.

14. Portelance L, Chao KS, Grigsby PW, et al. Intensity-modulated radiation therapy (IMRT) reduces small bowel, rectum, and bladder 
doses in patients with cervical cancer receiving pelvic and paraaortic irradiation. Int J Radiat Oncol Biol Phys 2001;51:261-266.

15. Roeske JC, Mundt AJ, Halpern $\mathrm{H}$, et al. Late rectal sequelae following definitive radiation therapy for carcinoma of the uterine cervix: a dosimetric analysis. Int J Radiat Oncol Biol Phys 1997;37:351-358.

16. Roeske JC, Lujan A, Rotmensch J, et al. Intensity-modulated whole pelvic radiation therapy in patients with gynecologic malignancies. Int J Radiat Oncol Biol Phys 2000;48:1613-1621.

17. Lujan AE, Mundt AJ, Yamada SD, et al. Intensity-modulated radiotherapy as a means of reducing dose to bone marrow in gynecologic patients receiving whole pelvic radiotherapy. Int $\mathrm{J}$ Radiat Oncol Biol Phys 2003;57:516-521.

18. Ahamad A, D'Souza W, Salehpour M, et al. Intensity-modulated radiation therapy after hysterectomy: comparison with conventional treatment and sensitivity of the normal-tissue-sparing effect to margin size. Int J Radiat Oncol Biol Phys 2005;62:1117-1124.

19. Ahmed RS, Kim RY, Duan J, et al. IMRT dose escalation for positive para-aortic lymph nodes in patients with locally advanced cervical cancer while reducing dose to bone marrow and other organs at risk. Int J Radiat Oncol Biol Phys 2004;60:505-512.

20. Mundt AJ, Roeske JC, Lujan AE, et al. Initial clinical experience with intensity-modulated whole-pelvis radiation therapy in women with gynecologic malignancies. Gynecol Oncol 2001;82:456-463.

21. Mundt AJ, Lujan AE, Rotmensch J, et al. Intensity-modulated whole pelvic radiotherapy in women with gynecologic malignancies. Int J Radiat Oncol Biol Phys 2002;52:1330-1337.

22. Brixey CJ, Roeske JC, Lujan $\mathrm{AE}$, et al. Impact of intensitymodulated radiotherapy on acute hematologic toxicity in women with gynecologic malignancies. Int J Radiat Oncol Biol Phys 2002;54:1388-1396.

23. Mundt AJ, Mell LK, Roeske JC. Preliminary analysis of chronic gastrointestinal toxicity in gynecology patients treated with intensity-modulated whole pelvic radiation therapy. Int J Radiat Oncol Biol Phys 2003;56:1354-1360.

24. Salama JK, Mundt AJ, Roeske J, et al. Preliminary outcome and toxicity report of extended-field, intensity-modulated radiation therapy for gynecologic malignancies. Int J Radiat Oncol Biol Phys 2006;65:1170-1176.

25. Beriwal S, Gan GN, Heron DE, et al. Early clinical outcome with concurrent chemotherapy and extended-field, intensity-modulated radiotherapy for cervical cancer. Int J Radiat Oncol Biol Phys 2007;68:166-171.

26. Gerszten K, Colonello K, Heron DE, et al. Feasibility of concurrent cisplatin and extended-field radiation therapy (EFRT) using intensity-modulated radiotherapy (IMRT) for carcinoma of the cervix. Gynecol Oncol 2006;102:182-188.
27. Buchali A, Koswig S, Dinges $S$, et al. Impact of the filling status of the bladder and rectum on their integral dose distribution and the movement of the uterus in the treatment planning of gynaecological cancer. Radiother Oncol 1999;52:29-34.

28. Han $\mathrm{Y}$, Shin EH, Huh SJ, et al. Interfractional dose variation during intensity-modulated radiation therapy for cervical cancer assessed by weekly CT evaluation. Int J Radiat Oncol Biol Phys 2006;65:617-623.

29. Lim K, Kelly V, Stewart J, et al. Pelvic radiotherapy for cancer of the cervix: is what you plan actually what you deliver? Int J Radiat Oncol Biol Phys 2009;74:304-312.

30. Chan P, Dinniwell R, Haider MA, et al. Inter- and intrafractional tumor and organ movement in patients with cervical cancer undergoing radiotherapy: a cinematic-MRI point-of-interest study. Int J Radiat Oncol Biol Phys 2008;70:1507-1515.

31. van de Bunt L, Jurgenliemk-Schulz IM, de Kort GA, et al. Motion and deformation of the target volumes during IMRT for cervical cancer: what margins do we need? Radiother Oncol 2008;88:233240.

32. Lee CM, Shrieve DC, Gaffney DK. Rapid involution and mobility of carcinoma of the cervix. Int J Radiat Oncol Biol Phys 2004;58:625-630.

33. van de Bunt $\mathrm{L}$, van der Heide UA, Ketelaars M, et al. Conventional, conformal, and intensity-modulated radiation therapy treatment planning of external beam radiotherapy for cervical cancer: the impact of tumor regression. Int J Radiat Oncol Biol Phys 2006;64:189-196.

34. Beadle BM, Jhingran A, Salehpour M, et al. Cervix regression and motion during the course of external beam chemoradiation for cervical cancer. Int J Radiat Oncol Biol Phys 2009;73:235-241.

35. Jhingran A, Winter K, Portelance L, et al. A phase II study of intensity modulated radiation therapy (IMRT) to the pelvic for post-operative patients with endometrial carcinoma (RTOG 0418). Int J Rad Oncl Biol Phys 2008;72:S16.

36. Lim K, Small W Jr, Portelance L, et al. Consensus guidelines for delineation of clinical target volume for intensity-modulated pelvic radiotherapy for the definitive treatment of cervix cancer. Int J Radiat Oncol Biol Phys, in press.

37. Mell LK, Kochanski JD, Roeske JC, et al. Dosimetric predictors of acute hematologic toxicity in cervical cancer patients treated with concurrent cisplatin and intensity-modulated pelvic radiotherapy. Int J Radiat Oncol Biol Phys 2006;66:1356-1365.

38. Mell LK, Tiryaki H, Ahn KH, et al. Dosimetric comparison of bone marrow-sparing intensity-modulated radiotherapy versus conventional techniques for treatment of cervical cancer. Int J Radiat Oncol Biol Phys 2008;71:1504-1510. 


\section{CME Activity: Use of Intensity-Modulated Radiation Therapy for Cervical Cancer}

To obtain credit, you should first read the journal article. After reading the article, you should be able to answer the following, related, multiple-choice questions. To complete the questions and earn continuing medical education (CME) credit, please go to www.medscapecme.com/journal/jnccn.

Credit cannot be obtained for tests completed on paper, although you may use the worksheet below to keep a record of your answers. You must be a registered user on Medscape.com. If you are not registered on Medscape.com, please click on the New Users: Free Registration link on the left hand side of the website to register.

Only one answer is correct for each question. Once you successfully answer all post-test questions you will be able to view and/or print your certificate.
For questions regarding the content of this activity, contact the accredited provider, CME@medscape.net. For technical assistance, contact CME@webmd.net.

American Medical Association's Physician's Recognition Award (AMA PRA) credits are accepted in the US as evidence of participation in CME activities. For further information on this award, please refer to http://www.ama-assn.org/ama/pub/category/2922. html. The AMA has determined that physicians not licensed in the US who participate in this CME activity are eligible for AMA PRA Category 1 Credits $^{\mathrm{TM}}$. Through agreements that the AMA has made with agencies in some countries, AMA PRA credit is acceptable as evidence of participation in CME activities. If you are not licensed in the U.S. and want to obtain an AMA PRA CME credit, please complete the questions online, print the certificate and present it to your national medical association.
1. On the basis of the above review by Drs. Loiselle and Koh, which of the following statements about potential pros and cons of IMRT is most correct?
A. Very few practicing radiation oncologists use IMRT
B. IMRT can increase dose to cancer targets while sparing adjacent healthy tissue
C. In posthysterectomy patients, IMRT vs conventional RT does not reduce gastroin- testinal toxicity

D. IMRT vs conventional RT does not reduce hematologic toxicity

2. Your patient is a 53-year-old woman who has undergone hysterectomy for cervical cancer. On the basis of the above review, which of the following technical strategies to improve the therapeutic ratio of IMRT would most likely be applicable to her management?
A. Portal imaging can easily show the entire ir- radiated volume
B. Quality-assurance measurements are not re- quired
C. IMRT is subject to inadvertent inadequate dose coverage or overdose caused by target motion or suboptimal target delineation
D. Inverse planning techniques allow the phy- sician's judgment to control dose delivery

3. According to the above review, in which of the following clinical scenarios is IMRT most likely to be indicated?
A. Dose escalation to para-aortic nodes and bulky sidewall disease
B. Cervical cancer in patients with an intact uterus
C. As a routine substitute for brachytherapy
D. Only in patients who have not previously received radiation therapy

\section{Activity Evaluation}

1. The activity supported the learning objectives. $\begin{array}{lccrr}\text { Strongly Disagree } & & \text { Strongly Agree } \\ 1 & 2 & 3 & 4 & 5\end{array}$

2. The material was organized clearly for learning to occur.
Strongly Disagree
1
2
3

3. The content learned from this activity will impact my practice.

Strongly Disagree

$\begin{array}{lllll}1 & 2 & 3 & 4 & 5\end{array}$

4. The activity was presented objectively and free of commercial bias.

Strongly Disagree Strongly Agree

$\begin{array}{lllll}1 & 2 & 3 & 4 & 5\end{array}$

To obtain credit, visit Medscape online at http://www.medscapecme.com/journal/jnccn. 